\title{
Narrowing the treatment gap in managing opioid use disorder
}

\author{
Joseph H. Donroe MD, Jeanette M. Tetrault MD
}

— Cite as: CMAJ 2018 March 5;190:E236-7. doi: 10.1503/cmaj.180209

See related article at www.cmaj.ca/lookup/doi/10.1503/cmaj.170958

$\mathbf{N}$ orth America is in the midst of a public health crisis related to opioid use disorder and overdose. In the United States, opioid overdose is the number-one cause of death from unintentional injuries, surpassing deaths related to motor vehicle injuries and exceeding the death toll seen at the peak of the HIV epidemic. ${ }^{1}$ Canada is second only to the United States in per-capita opioid use, and the numbers of opioidrelated deaths, emergency department visits and hospital admissions are climbing. ${ }^{2}$ Both countries are witnessing an influx in highly potent illicit synthetic opioids, such as fentanyl and carfentanil. Against this backdrop, the Canadian Research Initiative in Substance Misuse (CRISM) has published an evidencebased guideline on the management of opioid use disorder. ${ }^{3}$ This timely publication offers succinct guidance for physicians about the pharmacologic management of patients with opioid use disorder, and several aspects are worth highlighting.

Prior published guidelines on management of opioid use disorder have limited their target audience to clinicians involved in the evaluation and treatment of patients with the disorder, and to those providing psychosocially assisted pharmacologic treatment of opioid use disorder. ${ }^{4,5}$ However, at a time when fewer than one in five people who need treatment for opioid use disorder receive it, ${ }^{6}$ the scope of opioid guidelines should be expanded. The current Canadian guideline moves the narrative in the correct direction by clearly stating that it is intended for use by physicians and nurse practitioners, allied health care providers, pharmacists, medical educators and clinical managers, "with or without specialized experience in addiction treatment." "3

Opioid use disorder, like other substance use disorders, is prevalent, undertreated and often presents initially to general medical settings rather than to specialty settings. Available evidence shows the tremendous effect that primary care-led treatment can have on patient care outcomes, including mortality. ${ }^{7,8}$ The necessary expansion of access to care for opioid use disorder requires primary care providers to take the lead, as they do with other chronic diseases of similar complexity, such as diabetes. Primary care providers are trained to manage complex diseases, to understand the biopsychosocial factors contributing to illness and to know when referral to specialty care is needed. Most who specialize in the field of addiction

\section{KEY POINTS}

- A new Canadian guideline on the management of opioid use disorder is appropriately geared toward front-line health care providers, who are vitally important to decreasing the existing gap in treatment for opioid use disorder.

- Pharmacotherapy is unequivocally the evidence-based first-line treatment for opioid use disorder.

- Providers who are less experienced with buprenorphine induction may benefit from reading beyond the guideline or mentorship from an experienced clinician, particularly to avoid inadvertently precipitating uncomfortable opioid withdrawal.

- In select patients, it may be more appropriate to provide treatment with methadone from the outset.

medicine would agree that our expertise is needed in some complex cases, but the treatment of many patients with opioid use disorder can be managed successfully by well-informed primary care providers. Educational and treatment models that include mentoring for front-line providers who care for patients with opioid use disorder are available and effective. ${ }^{9,10}$ Barriers to primary care management of these patients go beyond the technical knowledge gaps addressed in the Canadian guideline, however, and include stigma, perceptions of complexity, time limitations in busy practices, concern about the need for additional onsite resources, and regulations on prescribing opioid agonist medication in some jurisdictions.

This Canadian guideline provides a simplified approach with the need for few additional resources, appropriately making the content accessible to front-line providers. ${ }^{3}$ The messaging is clear regarding several key elements of managing opioid use disorder for the target audience. First, pharmacotherapy is unequivocally the evidence-based first-line treatment for opioid use disorder. This is supported by countless well-designed studies showing the benefits to mortality and morbidity of pharmacotherapy, and the superiority of pharmacotherapy when compared with abstinencebased approaches. ${ }^{11,12}$ of the available pharmacologic options, buprenorphine should be considered the first-line agent for most front-line providers, given the dosing flexibility and improved safety profile compared with full opioid agonists. 
Second, withdrawal management alone should be avoided and is associated with relapse, morbidity and death. This is particularly relevant for front-line providers working in hospital settings where initiating pharmacotherapy for patients with opioid use disorder and connecting them to longitudinal care at the time of discharge can reduce discharges against medical advice and improve rates of hospital follow-up and treatment retention. ${ }^{13}$

Finally, the guideline emphasizes that psychosocial interventions should not be viewed as a mandatory component of treatment. Although psychosocial interventions are helpful for certain patients, and offering these services in an integrated model perhaps represents "ideal care," it can also serve as a logistical barrier to primary care providers.

There are some important limitations that providers should consider when implementing these recommendations. The title, "Management of Opioid Use Disorders: A National Clinical Practice Guideline," suggests the intention of a comprehensive set of recommendations. These recommendations, however, are specific to the pharmacologic management of opioid use disorder and do not include sufficient guidance on other management topics germane to front-line providers, such as screening, assessment, interdisciplinary treatment planning and integrating harm-reduction interventions. The process of buprenorphine induction also merits more thorough discussion, particularly for providers with less experience. Inadvertently precipitating withdrawal is not only uncomfortable to patients, but also may lead to discontinuation of care and the misperception that buprenorphine is not an effective medication. Additionally, whereas buprenorphine is the medication of choice for front-line providers, some patients would be more appropriately treated from the outset with methadone, which remains a life-saving medication, or switched to methadone after initial treatment with buprenorphine. More guidance on identifying such patients would have been useful. Finally, although the guideline suggests maintenance treatment with oral opioid antagonists as an adjunctive medication to promote abstinence, emerging international literature supports the effectiveness of extended-release depot naltrexone (though not currently available in Canada) compared with buprenorphine. ${ }^{14}$

Overall, this national guideline describing the pharmacologic management of opioid use disorder is timely and needed to address the expanding epidemic of opioid use disorder and overdose. Importantly, the guideline is geared toward front-line providers, who are vitally important to decrease the existing treatment gap. The critical next steps, at a national and international level, are to increase provider education in recognition and management of opioid use disorder and chronic pain, to reduce stigma associated with substance use disorders, to decrease provider barriers to prescribing opioid agonist treatment, to improve medication prescription coverage thereby increasing access to care, to expand access to harm-reduction modalities, and to encourage ongoing research into best practices for the prevention and treatment of opioid use disorder.

\section{References}

1. Ahmad FB, Rossen LM, Spencer MR, et al. Provisional drug overdose death counts. Hyattsville (MD): National Center for Health Statistics; 2018. Available: www.cdc.gov/nchs/nvss/vsrr/drug-overdose-data.htm (accessed 2018 Feb. 18).

2. Canadian Institute for Health Information; Canadian Centre on Substance Abuse. Hospitalizations and emergency department visits due to opioid poisoning in Canada. Ottawa: Canadian Institute for Health Information; 2016. Available: https://secure.cihi.ca/free_products/Opioid\%20Poisoning\%20Report \%20\%20EN.pdf (accessed 2018 Feb. 16).

3. Bruneau J, Ahamad K, Goyer M-È, et al. Management of opioid use disorders: a national clinical practice guideline. CMAJ 2018;190:E247-57.

4. Kampman K, Jarvis M. American Society of Addiction Medicine (ASAM) national practice guideline for the use of medications in the treatment of addiction involving opioid use. J Addict Med 2015;9:358-67.

5. Guidelines for the psychosocially assisted pharmacological treatment of opioid dependence. Geneva: World Health Organization; 2009. Available: https://www.ncbi .nlm.nih.gov/books/NBK143185 (accessed 2018 Feb. 17).

6. Wu L-T, Zhu H, Swartz MS. Treatment utilization among persons with opioid use disorder in the United States. Drug Alcohol Depend 2016;169:117-27.

7. Dupouy J, Palmaro A, Fatséas M, et al. Mortality associated with time in and out of buprenorphine treatment in French office-based general practice: a 7-year cohort study. Ann Fam Med 2017;15:355-8.

8. Lucas GM, Chaudhry A, Hsu J, et al. Clinic-based treatment of opioid-dependent HIV-infected patients versus referral to an opioid treatment program: a randomized trial. Ann Intern Med 2010;152:704-11.

9. Egan JE, Casadonte P, Gartenmann T, et al. The Physician Clinical Support SystemBuprenorphine (PCSS-B): a novel project to expand/improve buprenorphine treatment. J Gen Intern Med 2010;25:936-41.

10. Komaromy M, Duhigg D, Metcalf A, et al. Project ECHO (Extension for Community Healthcare Outcomes): a new model for educating primary care providers about treatment of substance use disorders. Subst Abus 2016;37:20-4.

11. Sordo L, Barrio G, Bravo MJ, et al. Mortality risk during and after opioid substitution treatment: systematic review and meta-analysis of cohort studies. BMJ 2017;357:j1550.

12. Mattick RP, Breen C, Kimber J, et al. Methadone maintenance therapy versus no opioid replacement therapy for opioid dependence. Cochrane Database Syst Rev 2009;(3):CD002209.

13. Donroe JH, Holt SR, Tetrault JM. Caring for patients with opioid use disorder in the hospital. CMAJ 2016;188:1232-9.

14. Lee JD, Nunes EV Jr, Novo P, et al. Comparative effectiveness of extendedrelease naltrexone versus buprenorphine-naloxone for opioid relapse prevention (X:BOT): a multicentre, open-label, randomised controlled trial. Lancet 2018;391:309-18.

\section{Competing interests: None declared}

This article was solicited and has not been peer reviewed.

Affiliation: Department of General Internal Medicine, Yale School of Medicine, New Haven, Conn.

Contributors: Both authors wrote the article, gave final approval of the version to be published and agreed to be accountable for all aspects of the work.

Correspondence to: Joseph Donroe, joseph.donroe@yale.edu 